\title{
A catalogue of X-ray bursts detected by JEMX onboard INTEGRAL
}

\author{
Celia Sánchez-Fernández* \\ INTEGRAL Science Operations Center (ISOC) \\ European Space Agency (ESA) \\ European Space Astronomy Centre (ESAC) \\ P.O. Box, 78 \\ 28691 Villanueva de la Cañada, Madrid, Spain \\ E-mail: Celia.Sanchezesciops.esa.int \\ Erik Kuulkers \\ INTEGRAL Science Operations Center (ISOC) \\ European Space Agency (ESA) \\ European Space Astronomy Centre (ESAC) \\ P.O. Box, 78 \\ 28691 Villanueva de la Cañada, Madrid, Spain \\ E-mail: Erik.Kuulkers@sciops.esa.int
}

\begin{abstract}
The wide field of view of JEMX onboard INTEGRAL allows concurrent monitoring of several sources in a single pointing, thus allowing serendipitous detection of interesting events from any of these targets. Taking advantage of this capability, we have performed a systematic search of Type-I X-ray bursts in the light curves of known X-ray bursters. For this task, all the the public data in the INTEGRAL archive have been analyzed. We present here the preliminary results of this work. In total, 70000 science windows have been analyzed, allowing the detection of 2243 Type-I X-ray burst candidates.
\end{abstract}

8th INTEGRAL Workshop, The Restless Gamma-ray Universe-Integral2010, September 27-30, 2010

Dublin Ireland

\footnotetext{
${ }^{*}$ Speaker.
} 


\section{Introduction}

Type I X-ray bursts are thermonuclear explosions on the surface of weakly magnetized accreting neutron stars (NS) in Low-Mass X-ray Binary (LMXB) systems (see reviews in Lewin et al. 1993; Strohmayer \& Bildsten 2003). Type I X-ray bursts show a large variety in profiles, but generally they exhibit a fast rise (from less than a second to $10 \mathrm{~s}$ ) and a longer, usually exponential, decay, lasting seconds to minutes. The waiting time between successive bursts can be regular or irregular, but typically on time scales of hours to days. Type-I Bursts radiate X-ray spectra with black body shapes and temperatures up to $3 \mathrm{keV}$, that cool during the decay, while the size of the emitting region remains constant.

The Peak flux for very bright bursts can reach the Eddington Luminosity at the surface of the NS, at which point the outward radiation pressure equals (or exceeds) the gravitational force binding the outer layers of the accreted material. Such bursts (known as Photospheric Radius Expansion events, PRE) frequently exhibit a characteristic spectral evolution in the first few seconds, with a local peak in blackbody radius and at the same time a dip in color temperature, while the bolometric flux remains approximately constant. Measurement of the X-ray flux during the PRE episode allows the determination of the distance to the burst source (e.g. Basinska et al. 1984), as well as the mass and radius of the neutron star (e.g. Damen et al. 1990; Özel 2006).

The large Field of View provided by the instrument Joint European Monitor for X-rays (JEM$\mathrm{X}$ ) onboard INTEGRAL (4.8 deg diameter, Fully Illuminated) allows simultaneous monitoring of many sources of interest in a single pointing, thus increasing the probability of burst detection. JEM-X works in the soft energy domain (3.5-35 keV) where most of the burst energy is released. These capabilities, combined with the accumulated INTEGRAL exposure in the Galactic Center and plane, where most known X-ray bursters are located, allow the study of X-ray burst activity with a baseline not possible using dedicated pointed observations.

\section{Data analysis}

We have used all the publicly available JEMX data in the INTEGRAL archive, (up to revolution 810), to carry out a systematic search of Type-I X-ray bursts serendipitously detected during INTEGRAL observations. 70000 science windows were analyzed, $80 \%$ of which provided good data. Data were processed using single pointings, with typical durations of 1800-3600 s. $\sim 70000$ science windows were analyzed, $80 \%$ of which provided good data. Standard OSA 8.0 processing procedures were used, and light curves were extracted with 5-sec time resolution in the 3-25 keV band. Type-I X-ray burst candidates were identified using standard burst searching procedures: we looked for deviations (> $4.5 \sigma$ ) of the average count rate in the source light curve. Whenever this was the case, we checked if the flare decay matched the profile of an exponential function $\left(\mathrm{e}^{-t / t}\right)$.

For the confirmed detections, we derived the relevant burst parameters: peak count rate, efolding decay time, burst integrated count rate, and the source persistent emission by the time of burst detection. The recurrence times between consecutive bursts of a certain source were also determined. 


\section{Preliminary results}

We have detected $2243 \mathrm{X}$-ray burst candidates from $45 \mathrm{X}$-ray bursters. In this analysis X-ray bursts from the rapid burster are not considered. Bursts have been detected from persistent and transient sources, allowing the study of burst activity over several regimes of accretion onto the NS. The analysis of these data on a source by source basis is now in progress, but overall relations between the main burst parameters have been derived and are presented in Figure 1.

(a)

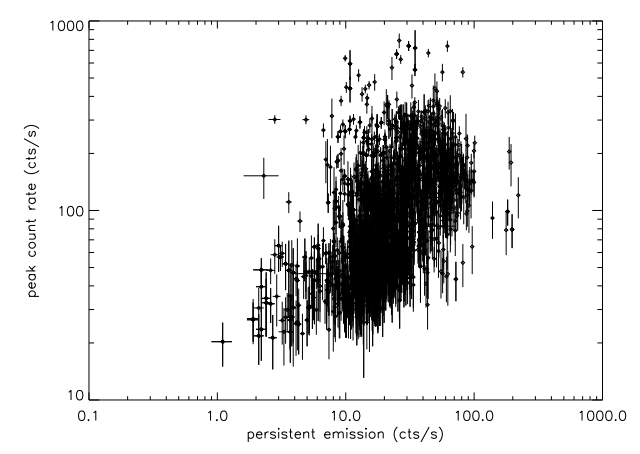

(c)

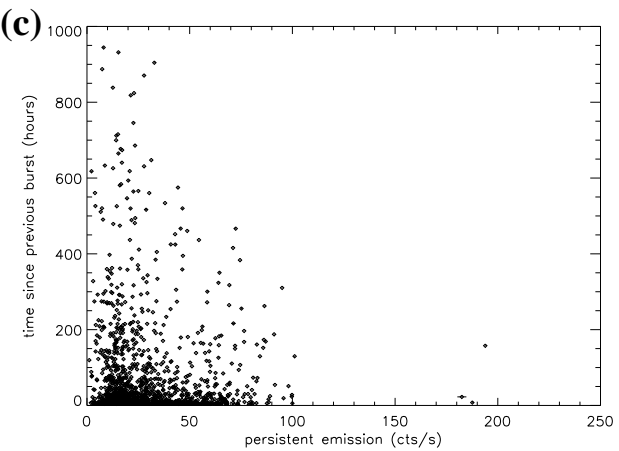

(b)

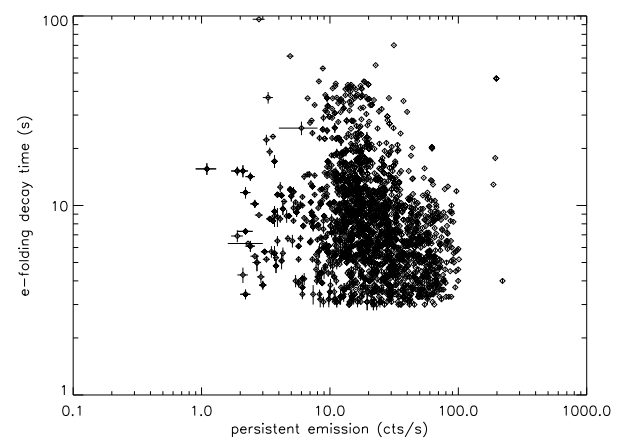

(d)

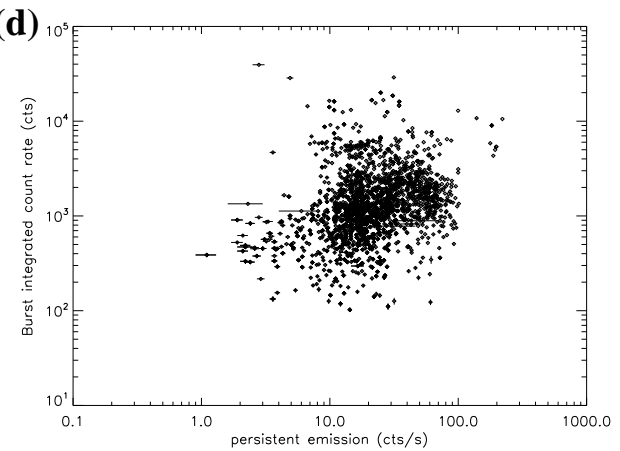

Figure 1: Using all the bursts detected in our sample, we have investigated the relations between the various burst parameters as measured from the burst light curve. The main correlations are displayed here. The burst peak count rate and burst integrated count rate (panes a and d) display a certain degree of correlation with burst persistent emission by the time of burst detection, while no evident correlation is found between the persistent emission of the system and the burst e-folding decay time, or burst waiting time and persistent emission (panels b and c). More conclusive data are expected when this analysis is performed on a source by source level.

\section{A case example: the $X-$ ray burster $4 U$ 1608-522}

$4 \mathrm{U} 1608-522$ is (recurrent) transient atoll system, which experiences outbursts that, in some cases can reach peak intensities of the order of the Crab (Gottwald et al, 1987). The source is also a known X-ray burster, displaying Type I X-ray bursts (Galloway et al, 2008 and references therein) as well as one superburst (Keek et al. 2008). In several occasions, burst oscillations at $619 \mathrm{~Hz}$ were measured, making this source a member of the group of rapidly spinning NS LMXB. 


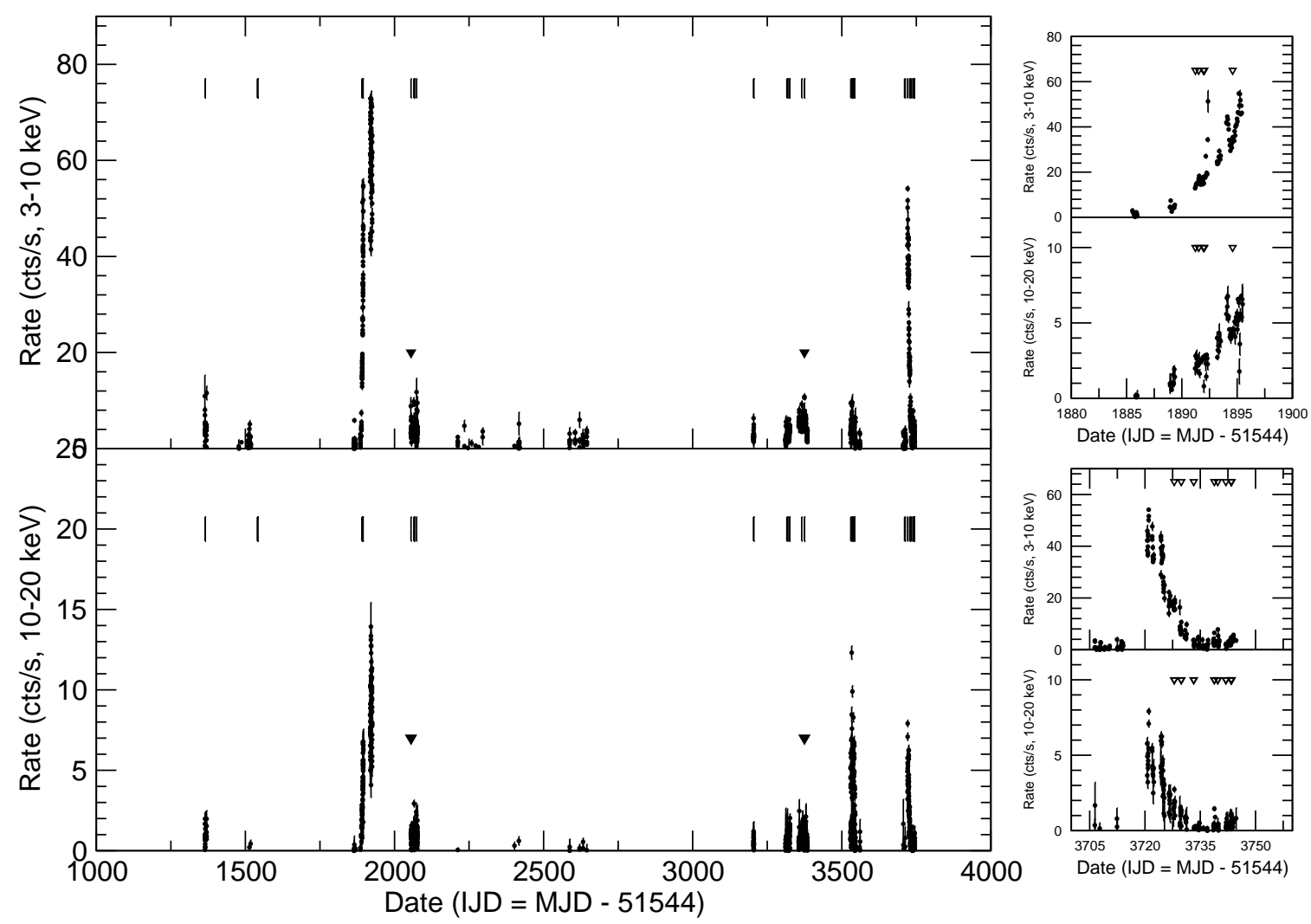

Figure 2: Left panel: The light curve of 4 U 1608-522 as seen by JEMX onboard INTEGRAL in the 3-10 $\mathrm{keV}$ band (upper panel and 10-20 keV) lower panel. The times of dection of X-ray bursts are marked by the down triangles superposed to the light curves. The time of occurrence of double/triple bursts are marked in red. Closer views of the outburst rise and decline are shown in the right panels of the figure.

The combination of propietary and archive data on this source, allowed us to monitor the bursting acitivity of 4U 1608-522 over all the INTEGRAL lifetime. We summarize here some of the results derived from this analysis. Further details are provided in Sanchez-Fernandez et al. 2011.

The light curve of $4 \mathrm{U} 1608-522$ as observed by JEMX over 8 years of observations is displayed in Figure 2. We have detected 38 type-I X-ray bursts from the system, at various accretion rates, ranging from quasi-quiescence to the higher levels of luminosity during outburst maximum. The times of occurence of type I X-ray bursts are marked by with vertical lines in Figure 2 .

\subsection{Double and triple bursts}

We have measured recurrence times between successive (double and triple, Figure 3) X-ray bursts as short as 10 minutes. These times of detection of these events are marked by the down triangles in Figure 2. It is worth to note here that both, the double and triple sequences of bursts happened at the lowest accretion rates. The short recurrence times between sucessive bursts do not allow for the accretion of the fuel that is burned during the second (or third) burst, indicating that most of the material burnt during the second (or third) burst was accreted before the first burst 


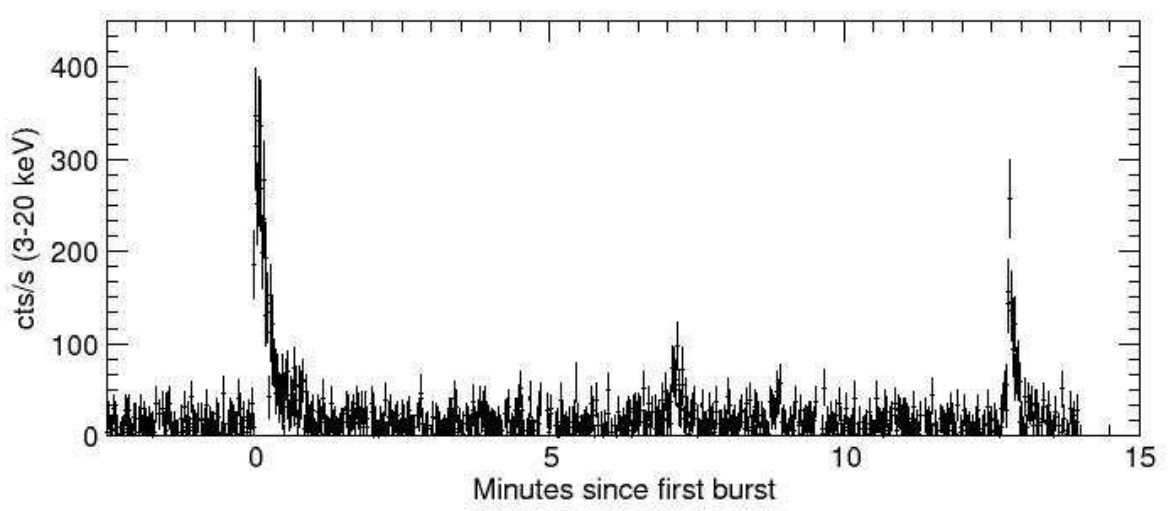

Figure 3: Triple burst from 4U 1608-522 observed by JEM-X on IJD=3374 (3-25 keV). Time resolution is $1 \mathrm{~s}$. The short recurrence times between sucessive bursts do not allow for the accretion of the fuel that is burned during the second (or third) burst. Instead, most of the material burnt during these bursts must be accreted before the first burst happened.

happened. This is in contradiction with current one-dimensional multi-zone models, which predict that during a flash over $90 \%$ of the available fuel is burned (e.g., Woosley et al. 2004), and requires further analysis and revision of current models.

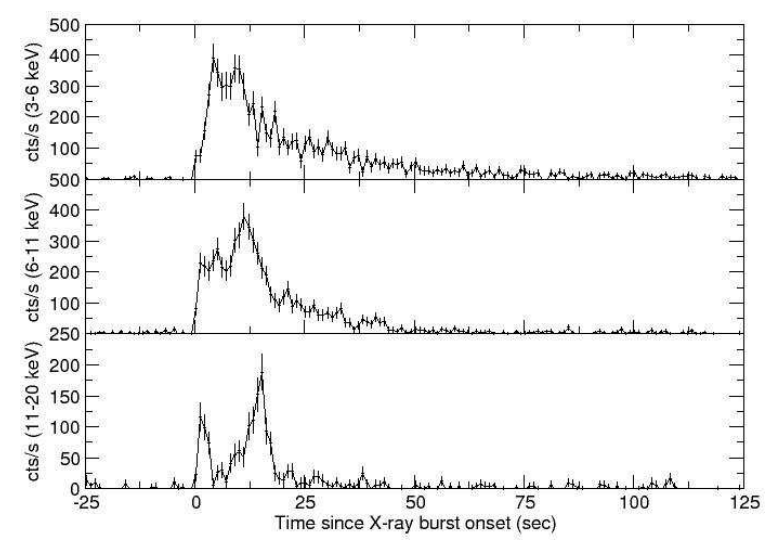

Figure 4: Light curve of the PRE event detected on IJD=3323, displaying the characteristic PRE profile. The expansion of the atmosphere, while the bolometric flux remains approximately constant leads to a dip in color temperature. In some cases, the temperature may become so low that the peak of the radiation shifts to the lower energies, even to the UV, leaving the drop observed in the burst light curve. The effect is more evident at higher energies. Time resolution is $1 \mathrm{sec}$.

\subsection{PRE bursts}

Photospheric radius expansion was detected in some of the bursts, for which the light curve at higher energies displayed the characteristic profile of a PRE burst: when the expansion is large, the temperature may become so low that the peak of the radiation shifts to the lower energies, even 
to the UV, leaving the drop observed in the burst light curve. Detailed spectral analysis of these events is still in progress.

\section{Conclusions}

We have developed a burst searching procedure which has been sucessfully applied to the analysis of the available public data in the INTEGRAL archive. With this procedure, 2243 Type-I X-ray burst candidates have been found from $45 \mathrm{X}$-ray bursters. Detailed analysis of these data on a source by source basis is still in progress.

\section{References}

[1] E. M. Basinksa et al, 1984, ApJ 281, 337.

[2] E. Damen et al, 1990, A\&A, 237, 103.

[3] D. K. Galloway et al. 2008, ApJS, 179, 360.

[4] W. H. G. Lewin et al, 1993, SpScRev, 62, 223.

[5] F. Özel, F., 2006, Natur, 441, 1115.

[6] Sanchez-Fernandez et al, in preparation.

[7] T. Strohmayer, L. Bildsten, 2006, in Compact Stellar X-Ray Sources, CUPress, 113.

[8] S. E. Woosley, \& E. E. Taam, 1976, Nature, 263, 101. 